\title{
ACOUSTIC DOUBLE REFRACTION IN LOW-POROSITY ROCKS
}

\author{
By Terry Todd, Gene Simmons, and W. Scott Baldridge
}

\begin{abstract}
Anisotropy in physical properties of rocks can arise from preferred mineral orientation, mineral layering, nonhydrostatic stress, and anisotropic crack distribution. For instance, all of the following cause acoustic double refraction: preferential orientation of olivine grains in dunites, alternating layers in laboratory-sized samples of such mineral pairs as olivine-feldspar, wollastonite-diopside, and garnetpyroxene, alternating layers of basalt flows and lunar breccias, anisotropy in crack distribution of most granites, and anistropy in crack distribution induced by uniaxial stress. We discuss, both experimentally and theoretically, shear-wave propagation in these rock types and indicate how the laboratory data may be applied to the interpretation of the anisotropy observed in the Earth's crust and upper mantle. We discuss the possibility of elastic anisotropy in the Moon.
\end{abstract}

\section{INTRODUCTION}

Values of physical properties of rocks at effective pressures below a few kilobars are determined primarily by mineral content and the presence or absence of microcracks. Preferred orientation of microcracks or individual minerals causes anisotropy in physical properties. Mineral anistropy can be caused by alignment of individual grains or layering of one or more minerals. Crack anisotropy can be naturally occurring or artificially induced. The phenomenon of anistropy can be used to improve our understanding of the Earth's crust and upper mantle through a study of physical properties which are sensitive to mineral orientation and crack distribution. Elastic properties, i.e., shear and compressional velocities, which can readily be measured in situ, are such physical properties.

Thill et al. (1969) have shown that compressional-wave velocity is a particularly sensitive indicator of mineral anistropy and crack distribution in $d r y$ low-porosity rocks. They found that velocities in the Yule marble were slowest parallel to a direction of distinct preferred orientation of optic [0001] axes of calcite, the slow velocity direction in single crystal calcite, and that velocities in the Salisbury granite were lowest for a direction normal to a plane containing a dense concentration of cracks in quartz grains. However, there are certain inherent problems in the use of their data to interpret compressional velocities of rocks in situ. For instance, the compressional velocity of lowporosity rocks when saturated is typically 20 to 30 per cent greater than the same rocks when completely dry (Nur and Simmons, 1969b). The degree of saturation in situ is generally not known but is probably near 100 per cent. If saturation is complete, pore pressure effects (Todd and Simmons, 1972) must be considered. In addition, in order to determine anisotropy with compressional waves, two perpendicular measurements of compressional velocity are required. The two waves travel different paths, and problems arise involving compositional variations between the two propagation paths. It would be advantageous to measure some physical property which would provide directional information without having to cope with local values of, and local variations in, the percentage of saturation, pore pressure, and composition.

For a particular direction of propagation in elastically anisotropic media, there can exist a quasi-longitudinal wave and two quasi-shear waves. Particle motions of the three 
waves are perpendicular to each other and generally neither parallel nor perpendicular to the propagation direction. Although shear waves with high signal-to-noise ratios can readily be produced and monitored in laboratory experiments, shear waves with equally high signal-to-noise ratios are extremely difficult to obtain in the field. Shear waves, being late arrivals, are often masked by noise due to compressional waves and surface waves. However, when shear velocities can be measured in situ, they provide more information about anistropy than compressional-wave velocities. The velocity difference between vertically and horizontally polarized shear waves is explicitly related to the magnitude and symmetry of mineral and crack anisotropy. The degree of saturation has negligible effect on shear velocity at seismic frequencies (Nur and Simmons, 1969b). Therefore, the interpretation of shear data in terms of the intrinsic physical properties of the rock and crack distribution is the same regardless of the degree of saturation. Also, because only one propagation path is used, compositional variations along the path and the effect of pore pressure do not change the magnitude of the difference between the vertically and horizontally polarized waves. We conclude that shear-wave velocities of rocks in situ hold high potential for the study of rocks.

In laboratory studies, acoustic double refraction, or the arrival of two shear waves of different velocities, has previously been observed in single crystals (Simmons and Birch, 1963), in rocks with stress-induced anisotropy in crack distribution (Nur and Simmons, 1969a), and in rocks with anistropy in mineral orientation(Christensen and Ramananantoandro, 1971). In addition, Simmons (1964) and Tilman and Bennett (1972) report variations in shear velocity with orientation that exceed 10 per cent for several rocks, but they do not discuss the origin of the anistropy. We report new data for double refraction in a granite having naturally occurring crack anisotropy.

In situ double refraction has been observed in small scale refraction surveys over hundreds of meters (Jolly, 1956) and in large scale seismic studies over thousands of kilometers (Byerly, 1938). On the basis of the laboratory studies of acoustic double refraction, we suggest that in situ observations of double refraction can be used to study anisotropy in the Earth's crust and upper mantle and to determine the cause of anisotropy, mineral or crack.

\section{Single CRystals}

Consider a plane shear wave with known polarization $P$ incident on the surface of a single crystal with direction cosines $l_{1}, l_{2}$, and $l_{3}$. Wave propagation in the crystal is described by the Christoffel equation (Hearmon, 1961)

$$
\left|\Gamma_{i j}-\delta_{i j} \rho v^{2}\right|=0
$$

where $\Gamma_{i j}$, the Christoffel stiffnesses, are related to the elastic constants and depend on direction, $\rho$ is density, and $v$ is velocity. The solution of this cubic equation for a given direction shows that one compressional and two shear waves can propagate. Hence, the incident shear wave is resolved into two shear waves that travel along the same path with different velocities. The particle motions of the two waves are perpendicular to each other, but generally not perpendicular to the direction of propagation. The shear waves are quasi-shear waves, having distinct energy flux vectors and different velocities. The phenomenon is called acoustic double refraction (Waterman and Teutonico, 1957), and we will use the abbreviation ADR.

In their measurement of the elastic constants of the cubic crystal pyrite, Simmons and Birch (1963) observed ADR. A shear wave polarized in the (111) direction was transmitted along the (110) direction. If the displacement of the input wave were in the (001) or (110) 
direction, only a single shear arrival of velocity $\left(C_{44} / \rho\right)^{1 / 2}$ or $\left[\left(C_{11}-C_{12}\right) / 2 \rho\right]^{1 / 2}$, respectively, would be visible. However, at the intermediate $(\overline{1} 11)$ polarization, the motion of the input wave was resolved into the two components, recognized by Simmons and Birch as two distinct shear arrivals traveling with the above velocities and a series of reflections of the initial pulses.

Single crystal data can be used to illustrate the way in which seismic anisotropy in rocks can be studied. Consider wave propagation along the (110) direction of a cubic crystal. The velocities are

$$
\begin{aligned}
& V_{p}=\left[\frac{1 / 2\left(C_{11}+C_{12}\right)+C_{44}}{\rho}\right]^{1 / 2} \\
& V_{s 1}=\left[\frac{C_{44}}{\rho}\right]^{1 / 2} \\
& V_{s 2}=\left[\frac{C_{11}-C_{12}}{2 \rho}\right]^{1 / 2}
\end{aligned}
$$

and a measurement of the three velocities along this single direction is sufficient to determine the full set of elastic constants $\left(C_{11}, C_{12}\right.$, and $\left.C_{44}\right)$. Similarly, when shear waves propagate through rock, any anistropy causes a difference in the shear velocities. Measurement of shear velocities, therefore, gives information about the anistropy.

\section{ANISOTROPY IN Mineral OrIENTATION}

Anisotropy in the elastic properties of many igneous rocks is caused by a preferred orientation of individual mineral grains in the rocks (Birch, 1960 and Brace, 1965). For example, the intrinsic velocities of some ultra-basic rocks are influenced by a preferred orientation of olivine grains. Birch $(1960,1961)$ and Simmons (1964) noted anisotropies in compressional and shear velocities of dunites at high pressure that exceeded 10 per cent. Christensen (1966) and Crosson and Lin (1971) correlated this anisotropy with preferred orientation of olivine grains in two dunites, and Christensen (1971) showed that this anisotropy exists in situ throughout the Twin Sisters dunite in Washington State. Ernst (1935), Brothers and Rodgers (1969), and numerous other authors showed a similar preferred orientation of olivine fabric in many ultra-basic nodules. In addition, through a series of high-temperature compression tests on dunite, peridotite, and olivine powder, Ave'Lallement and Carter (1970), Carter et al. (1972), and Baker and Carter (1972), have produced samples experimentally which show preferred mineral orientation.

Acoustic double refraction, caused by mineral orientation, has been documented by Christensen and Ramananantoandro (1971) in a comprehensive study of the Twin Sisters dunite. Their fabric diagrams for the Twin Sisters dunite show a distinct concentration of olivine $\bar{a}$ axes, surrounded at $90^{\circ}$ by girdles of $\bar{b}$ and $\bar{c}$ axes. [Stonely (1949) termed this symmetry transverse anisotropy. For a comprehensive discussion of types of anisotropy in rocks see Paterson and Weiss (1961).] Due to the large directional variations in compressional and shear velocity in single crystal olivine (Verma, 1960), the anisotropy of the fabric in the Twin Sisters dunite causes acoustic double refraction.

The anisotropy and double refraction of the Twin Sisters dunite are attributed to mineral anisotropy because the anisotropy remains at high pressures of 5 to $10 \mathrm{~kb}$ where microcracks are closed. Christensen and Ramananantoandro (1971) illustrated acoustic double refraction in the Twin Sisters dunite by propagating a shear wave normal to the $\bar{a}$ axis, the fast compressional velocity direction, and rotating the polarization of the input 
and receiving transducers through a series of angles from parallel to the $\bar{a}$ axis $\left(0^{\circ}\right)$ to perpendicular to the $\bar{a}$ axis $\left(90^{\circ}\right)$. At $0^{\circ}$, one distinct shear arrival was evident. As the polarization of the transducers was rotated, the amplitude of this shear arrival decreased and reached zero at $90^{\circ}$; a second arrival of slower velocity was then evident. At intermediate orientations, both arrivals were visible but of lower amplitude. The double refraction is predicted from the theory of wave propagation in transversely isotropy elastic media.

Stoneley (1949) discussed wave propagation in a medium with transverse isotropy. The Christoffel equations are greatly simplified for this symmetry. Let the velocities be $V_{p}, V_{s 1}, V_{s 2}$, and let $\psi$ be the angle between the propagation direction and the symmetry axis. In Nur's (1971) notation, the velocities are

$$
\begin{aligned}
& V_{p}=\left[\frac{A+B}{2 \rho}\right]^{1 / 2} \\
& V_{s 1}=\left[\frac{A-B}{2 \rho}\right]^{1 / 2} \\
& V_{s 2}=\left[\frac{C_{1212} \sin ^{2} \psi+V_{1313} \cos ^{2} \psi}{\rho}\right]^{1 / 2}
\end{aligned}
$$

where

$$
\begin{aligned}
A= & C_{1111} \sin ^{2} \psi+C_{1313}+C_{3333} \cos ^{2} \psi \\
B= & \left\{\left(\left(C_{1111}-C_{1313}\right) \sin ^{2} \psi-\left(C_{3333}-C_{1313}\right) \cos ^{2} \psi\right]^{2}\right. \\
& \left.+4\left(C_{1133}+C_{1313}\right)^{2} \sin ^{2} \psi \cos ^{2} \psi\right\}^{1 / 2}
\end{aligned}
$$

and $\rho$ is density. For propagation along the symmetry axis $(\psi=0)$, the velocities are simply

$$
V_{p}=\left[\frac{C_{3333}}{\rho}\right]^{1 / 2}, \quad V_{s 1}=V_{s 2}=\left[\frac{C_{1313}}{\rho}\right]^{1 / 2}
$$

and for propagation normal to the symmetry axis

$$
V_{p}=\left[\frac{C_{1111}}{\rho}\right]^{1 / 2}, \quad V_{s 1}=\left[\frac{C_{1313}}{\rho}\right]^{1 / 2}, \quad V_{s 2}=\left[\frac{C_{1212}}{\rho}\right]^{1 / 2}
$$

For propagation normal to the concentration of $\bar{a}$ axes in olivine, equation (11) applies. We note explicitly that two distinct shear arrivals are predicted by the Christoffel equation for all directions of propagation other than along the symmetry axis.

\section{Geometrical Anisotropy-Mineral LAyERING}

A second source of anisotropy in igneous rocks is the arrangement of the individual minerals; the particular example of layering is most important in the Earth. For example, Uhrig and Van Melle (1955) found that the compressional velocity normal to interbedded layers of clastic and carbonate sediments was 10 to 20 per cent less than that along the layers. Backus (1962) showed that this anisotropy was consistent with the theory of wave propagation through layered media in which the wavelength is much greater than the layer thicknesses. Layering of rocks in situ include not only sedimentary rocks but also basalt flows interbedded with pyroclastics or soils, rhythmic layering of igneous bodies typified by the Palisades Sill described by Walker (1969), and compositional layering in metamorphic rocks. Large scale mineral layering is recognized in the Earth's crust as a 
$6-\mathrm{km} / \mathrm{sec}$ layer above a $7-\mathrm{km} / \mathrm{sec}$ layer separated by the Conrad discontinuity. Smallerscale mineral layering occurs as alternating layers in laboratory samples of such mineral pairs as olivine-feldspar, wollastonite-diopside, and garnet-pyroxene.

All rocks with mineral layering should exhibit double refraction. The difference between the two shear arrivals depends on the relative thickness of the layers with respect to wavelength and the properties of the individual layers.

The theory of wave propagation in layered media is well developed (Brekhovskikh, 1960 and Backus, 1962). The velocities of waves in layered media depend on several parameters, including the elastic properties of the minerals in each layer, layer thickness $(d)$, and the wavelength $(\lambda)$ of the propagating wave. For the short wavelength limit, $\lambda \ll a$, the velocities are simply time averages through the layers, and velocities in each layer can simply be calculated from the individual mineral elastic constants. In this case, $V_{s 1}=$ $V_{s 2}$. For the long wavelength limit $\lambda \gg d$, the solution is more complex, but has been solved by Backus (1962). The solution is similar to that for a transverse isotropic medium [equations (5), (6), and (7)] where the effective elastic constants involve averages over the elastic constants and densities of the individual layers. For propagation along the layers, two pseudo-shear waves are predicted. The velocity for polarization normal to the layering is always less than that for polarization parallel to the layering. For $\lambda \approx d$, the solution is extremely complex. Thomson (1950) gives the solution in terms of propagating matrices. Velocity solutions, requiring complete specification of layer thickness and boundary conditions, can be obtained numerically. Values of $V_{s 1}$ and $V_{s 2}$ are intermediate to those of the long and short wavelength limits. Two pseudo-shear waves of different velocity again exist.

\section{Naturally Occurring Crack Anisotropy}

The presence of microcracks in all low-porosity rocks significantly influences the values of elastic constants at low confining pressures (e.g., Adams and Williamson, 1923; Birch, 1960; Simmons, 1964; and Brace, 1965). An anisotropic distribution of microcracks generates anisotropy in values of effective elastic constants. Preferred orientations in crack distribution can result from primary features such as preferred mineral orientations (e.g., mica in a micaceous schist) where grain boundaries, cleavage, etc., are open cracks or from an anisotropic stress distribution acting on the rock after formation. For instance, because of extensive fracturing due to removal of overburden pressure by erosion, most granites are more intensely cracked in the horizontal plane than in any other plane (Jahns, 1943). The Chelmsford granite (Table 1) is a particularly good

TABLE 1

Description of Rock Samples of Chelmsford Granite

$\begin{array}{lr}\text { Modal analysis } & \text { (Percentage of volume): } \\ \text { Quartz } & 34.4 \\ \text { Microcline } & 36.3 \\ \text { Plagioclase } & 18.5 \\ \text { Muscovite } & 8.1 \\ \text { Biotite } & 0.9 \\ \text { Other } & 1.0 \\ \text { Density: } 2.63 \mathrm{~g} / \mathrm{cc}\end{array}$

Note: The modal analysis is an average from three thin sections oriented in the rift, grain, and headgrain (2,700 points each). 
example of both crack anisotropy resulting from stress fracturing and mineral orientation of mica. Peng and Johnson (1972) noted that cracks are preferentially oriented parallel to the horizontal rift plane $(\mathrm{R})$ relative to either the grain $(\mathrm{G})$ or headgrain $(\mathrm{H})$ planes. The rift, grain, and headgrain are quarrying terms used to describe the easiest, intermediate, and most difficult directions for splitting. In the Chelmsford granite, the rift is perpendicular to the grain, but the headgrain can be chosen at any angle relative to the rift or grain (Skehan et al. 1964). In our sample, the headgrain plane is normal to both the rift and grain planes. The intense cracking parallel to the rift is due to anisotropic stresses caused by the removal of overburden material by erosion. There are secondary, but much smaller, concentrations of cracks parallel to the grain and headgrain planes (Peng and Johnson, 1972). Splitting parallel to the grain plane is controlled by a concentration of

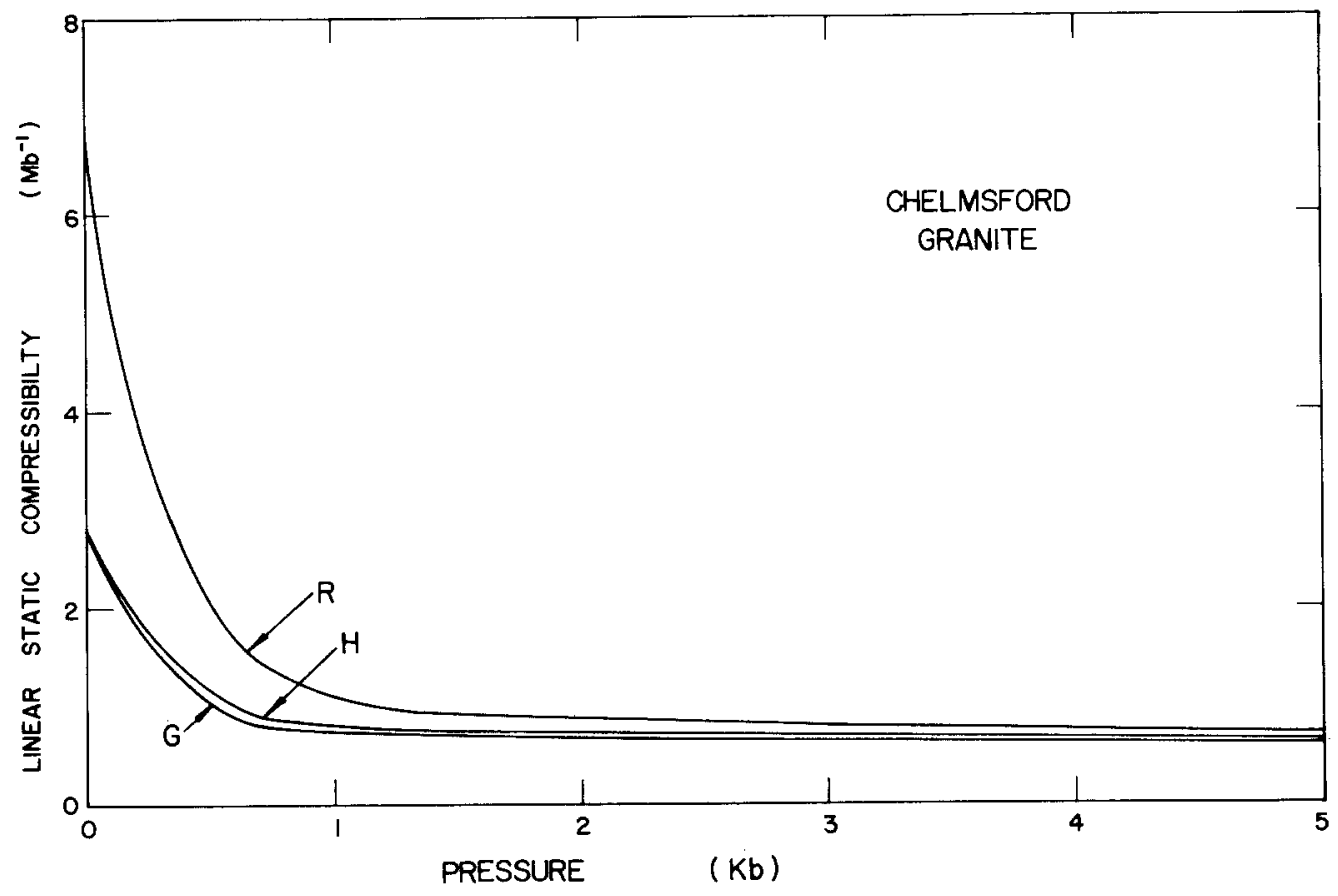

FIG. 1. Linear static compressibility $\left(M b^{-1}\right)$ of Chelmsford granite normal to the rift (R), grain (G), and headgrain $(\mathrm{H})$ planes.

microcracks lying between $7^{\circ}$ and $12^{\circ}$ of the grain plane (Peng and Johnson, 1972), a preferred orientation of mica (and thus cleavage cracks in mica) along the grain plane, and possible open cracks between mica flakes and adjacent minerals. A less pronounced concentration of cracks in the headgrain plane accounts for the ease of splitting along the grain surface relative to the headgrain surface.

The effect of crack anisotropy on the linear static compressibility of Chelmsford granite is illustrated in Figure 1. The sample used for these measurements was quarried about $50 \mathrm{ft}$ directly beneath, and oriented with respect to, that of Peng and Johnson. The larger number of cracks perpendicular to the rift is manifested by a much larger compressibility at low pressures (about 130 per cent at $P=0$ ). At high pressure, where cracks are closed, the three linear compressibilities are the same. Peng and Johnson noted similar behavior in the value of Young's modulus for the Chelmsford granite. 
An anisotropic crack distribution generates anisotropy in effective elastic constants and, hence, double refraction. The phenomenon of double refraction is illustrated in Figure 2 for the Chelmsford granite. A single sample was cored normal to the grain plane, the ends of the sample were ground parallel to $\pm 0.001 \mathrm{in}$, and the sample dried for $24 \mathrm{hr}$ at $70^{\circ} \mathrm{C}$ in a vacuum oven. The sample was then jacketed with thin copper foil which was seated tightly to the sample at a pressure of $5 \mathrm{~kb}$, and $\mathrm{AC}$ cut quartz transducers were fixed with epoxy to the copper end pieces. Shown in Figure 2 are a series of tracings of received shear waves that propagated perpendicular to the grain plane. Polarization of the transducers varied from normal to the headgrain plane $\left(0^{\circ}\right)$ to normal to the rift plane $\left(90^{\circ}\right)$. Because the propagation direction is normal to the grain plane and, hence, the concentration of mica flakes, shear waves of all polarizations are influenced in the same way by the mica. Thus, the only parameter influencing velocity differences is the anisotropy in crack distribution. The wave forms are shown at confining pressures of 50 , 250 , and 2,000 bars.

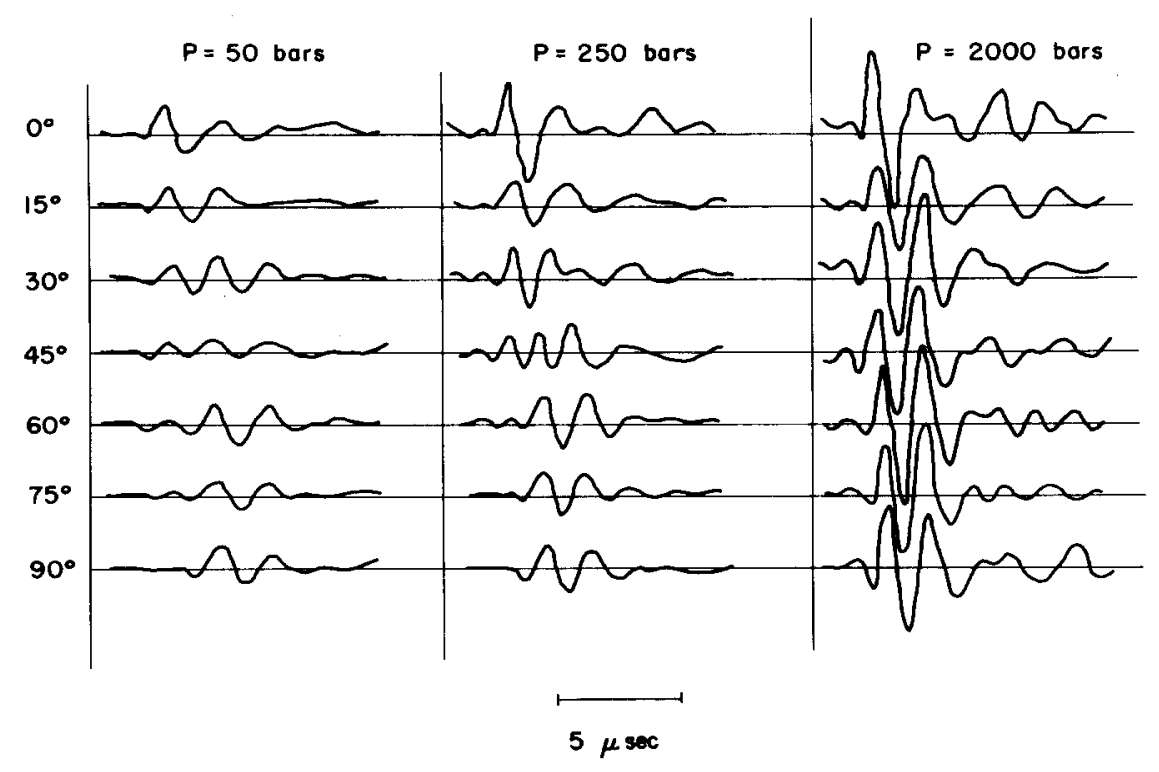

CHELMSFORD GRANITE

FIG. 2. Acoustic double refraction in Chelmsford granite. Tracings of shear arrivals are shown at 50,250 , and 2,000 bars for propagation normal to the grain, and transducer polarization at a series of angles between the normal to the headgrain $\left(0^{\circ}\right)$ and the normal to the rift $\left(90^{\circ}\right)$.

At low pressure, two distinct arrivals are evident, a fast arrival for transducer polarization normal to the headgrain $\left(0^{\circ}\right)$ and a slower arrival for transducer polarization normal to the rift $\left(90^{\circ}\right)$. Both arrivals are visible at intermediate angles. Because of the concentration of crack poles parallel to the rift, the shear velocity normal to the rift is less than that normal to the headgrain. Increasing the pressure to 250 bars closes some fraction of the cracks and reduces the velocity difference between the two shear waves. At 2,000 bars, most cracks are closed and double refraction is barely visible. At still higher pressures, only one shear wave exists at all angles, $0^{\circ}$ through $90^{\circ}$. Where all cracks are closed, anisotropy no longer exists, and the cause of double refraction is removed.

The effect of crack anisotropy on velocities has been treated theoretically by Nur (1971) by extending Walsh's (1965) concept of penny-shaped cracks to orientational 
distributions. Following the solutions given by Stoneley (1949) for a transverse isotropic medium, Nur obtained expressions similar to those given in equations (5), (6), and (7) with the important difference that now the effective elastic constants involve an integral tion over crack parameters. If the crack distribution is anisotropic, two pseudo-shear waves exist. The shear velocity is lowest for a direction normal to the greatest concentration of crack planes.

\section{Stress Induced Crack Anisotropy}

Crack anistropy can be induced in rocks containing microfractures by the application of a nonhydrostatic stress field. The resulting anisotropy in crack distribution causes anisotropy in physical properties. The variation in compressional velocity induced by uniaxial stress has been studied for concrete (Jones, 1952) and several low-porosity igneous rocks (Shimozuru, 1955; Tocher, 1957; Matsushima, 1960; Valarovich et al., 1963; Nur and Simmons, 1969a; and Thill, 1972). Cracks oriented normal to the stress axis are closed by a uniaxial stress; velocity, therefore, increases with stress until stresses near the fracture strength, $\mathrm{C}$, are reached. At stresses near $\mathrm{C}$, the large increase in crack density causes the velocity to decrease slightly. On the other hand, cracks aligned parallel to the stress axis are relatively unaffected by the axial stress until new cracks are formed at stress about $\mathrm{C} / 2$; velocities, therefore, only increase slightly with stress for stresses less than $\mathrm{C} / 2$. At stresses near $\mathrm{C} / 2$, for unconfined laboratory samples, either new cracks begin to form parallel to the stress axis or pre-existing cracks along the stress axis are opened further (Brace et al., 1966). Therefore, at stress greater than $\mathrm{C} / 2$, the velocity normal to the stress axis decreases steadily with increasing stress until the rock fractures. However, in the Earth, dilatancy probably cannot occur because of confining pressures. The anisotropy in stress values probably does not exceed 100 to 200 bars for tectonic regions (e.g., Wyss and Molnar, 1972; Aki, 1967). The critical observation is this: cracks normal to the stress axis close, but cracks parallel to the stress axis are relatively unaffected by uniaxial stress. Thus, velocities increase parallel to the axis but are relatively unaffected normal to the axis.

The effect on shear velocity of stress-induced anisotropy in crack distribution has been studied by Nur and Simmons (1969a). They used the Barre granite which is isotropic in both physical properties and crack distribution, even at low confining pressures. By applying a uniaxial stress, they created a plane of low crack density (transverse anisotropy). This symmetry is the same as that occurring naturally in the Chelmsford granite. Their observations of induced double refraction are therefore similar to those we have outlined above for the Chelmsford granite.

The theoretically predicted effect of stress on the elastic properties of rocks containing microcracks is included in Nur's (1971) paper. Nur has derived expressions for velocities under hydrostatic pressure, for velocities across axes of uniaxial stress, and for velocities along axes of uniaxial stress in the Barre granite, and has found that his theory adequately describes velocity variations under both hydrostatic pressure and uniaxial stress. In particular, the observed double refraction was well described by the theoretical expressions.

\section{In-SITU ANISOTROPY}

In-situ velocity anisotropy has previously been reported. Among the first to notice two distinct shear arrivals associated with horizontally $(S H)$ and vertically $(S V)$ polarized shear waves traveling through the interior of the Earth, were Neumann (1930) and 
Byerly (1934, 1938). For instance, for an earthquake occurring off the California coast in 1934, Byerley (1938) noted an $S V$ arrival approximately $14 \mathrm{sec}$ after the $S H$ arrival on a seismogram recorded in Massachusetts. This particular example of double refraction in situ is difficult to interpret in terms of anisotropy in the deep crust or upper mantle because the path of propagation is extremely complex. East-west variations in heat flow (Horai, 1969), velocity structure (Herrin, 1969), and seismic attenuation (Solomon, 1972) are evident across the continental block of North America; however, the differences in $S H$ and $S V$ arrival times do suggest that mineral anisotropy occurs in the upper mantle beneath the U.S. This fact is further substantiated by McEvilly (1964) and Brune (1969) who report anomalous low-phase velocities for Rayleigh waves $(S V)$ when compared with phase velocities for Love waves $(S H)$ in the central U.S.

In addition to anisotropy in continental structure, seismic anistropy has also been reported in oceanic areas. In a number of refraction surveys in the Pacific Basin, Hess (1964), Christensen (1969), Raitt et al. (1969), Raitt (1969), and Keen and Barret (1971) report azimuthal variations of 0.3 to $0.6 \mathrm{~km} / \mathrm{sec}$ in compressional velocity. They interpret this anisotropy in terms of preferred orientation of olivine fabric. However, they have not yet measured shear velocities. If anisotropy is present, it will cause double refraction. Shear velocities, therefore, provide an additional method for both verifying the existence of such anisotropy and interpreting the anisotropy in terms of the elastic properties of the upper mantle.

A particularly intriguing example of ADR has been reported by Bentley (1972). During an ultrasonic velocity-logging experiment to depths of $1,550 \mathrm{~m}$ in the Antarctic ice sheet, he noted anisotropies in compressional and shear velocities. He attributed the anisotropy to one, and in some cases two, directions of preferred orientation of the ice crystals. The alignment of ice crystals is a common phenomenon throughout the ice sheet in Antarctica (Bentley, 1972). Kamb (1972) has shown that the preferred orientation is stress induced.

There are several in situ observations of double refraction caused by anisotropy in mineral layering and crack distribution. For instance, Jolly (1956) has investigated the propagation of $S V$ and $S H$ waves through a uniform section of shale. The shale, having a dense concentration of horizontally oriented cracks, in addition to mineral layering, provides an ideal transverse isotropic medium. At shallow depths $(<100 \mathrm{~m})$ the $S V$ wave was 50 to 100 per cent slower than the $S H$ wave; the difference in the two shear velocities decreased with depth. Crack orientation and mineral layering evidently controlled the difference in the shear velocities at shallow depths.

Layering occurs on a larger scale across extensive areas of the Earth and lunar surfaces in the form of one or more layers of basalts or breccias. In earthquake seismology, one propagates shear waves of wavelength on the order of kilometers through the Earth and Moon. Shear waves traveling through layers of such thickness should exhibit double refraction. If the mare surface is covered with layered basalts and breccias, two shear arrivals should be evident on seismograms recorded from natural or man-made impacts on the lunar surface. Any observed ADR can be used to study layered structure on the lunar surface.

Because nonuniform stress causes anisotropy in crack distribution and, therefore, anisotropy in compressional velocity, Tocher (1957) suggested that compressional velocity measurements near fault zones can be used to monitor stress changes. Eisler (1969), Aki et al. (1970), and Kanamori (1970) have followed this suggestion and interpreted velocity changes in situ in terms of stress build up. However, because of the inherent problems, mentioned previously, in interpreting compressional velocity in terms of the degree of saturation, pore pressure, and composition, extreme caution must be taken in the interpretation of compressional data. 
We suggest that the key to observing crack closure under stress lies with shear velocity measurements at more than one polarization. For instance, a number of authors (Wyss and Molnar, 1972 and Aki, 1967) suggest that anisotropy in stress values in excess of 100 to 200 bars is unlikely in the crust. The stress drops associated with earthquakes induced by these stresses is one to two orders of magnitude less than the stresses. Using these stresses and the laboratory data of Nur and Simmons (1969a) for stress-induced shear velocity anisotropy in granite, we should expect shear velocity anisotropy of about $0.2 \mathrm{~km} / \mathrm{sec}$, or about 8 per cent difference in the two shear velocities at 200 bars. The corresponding velocity differences for two-compressional waves normal and parallel to the stress axis is 13 per cent at 200 bars for completely dry rocks. Although the $S H$ and $S V$ shear velocity measurements are not quite as sensitive to anisotropy as are the two perpendicular compressional velocity measurements, the difference between the shear velocities along a single propagation path carries only information about anisotropy. The difference in the $S H$ and $S V$ arrivals is not affected in any way by the saturation or compositional variation. Any anisotropy is due solely to the closing of cracks in the rock. A measurement of the difference between the $S H$ and $S V$ velocities near a source of stress build-up describes only the stress acting on the rock.

\section{Discussion}

In the laboratory, we have considered the effects of crack and mineral anisotropy on the propagation of shear waves through rocks on samples chosen specifically for their anisotropy. How do we apply the laboratory observations to the interpretation of anisotropy in situ? For instance, if anisotropy is present in both crack distribution and mineralogy, how do we separate the two sources of anisotropy? To answer these questions, we consider a simple example.

The Chelmsford granite contains both crack anisotropy (i.e., cracks preferentially oriented parallel to the rift) and mineral anisotropy due to mica orientation (parallel to the grain). Both of these sources of anisotropy influence shear velocities. In Figure 3 we have plotted the shear velocities for propagation perpendicular to the headgrain and polarization normal to the rift $\left(V_{S_{H R}}\right)$ and to the grain $\left(V_{S_{H G}}\right)$ planes. The value of $V_{S_{H R}}$ should be most sensitive to the intense concentration of cracks in the rift, the value of $V_{S_{H G}}$ should be sensitive to the mica concentration in the grain. At low pressures cracks are open, and the effect of the dense crack concentration in the rift dominates the effect of mica orientation; thus, $V_{S_{H R}}<V_{S_{H G}}$. At higher pressures the cracks are closed, and the effect of the intrinsically lower velocity perpendicular to the mica cleavage dominates that of cracks; thus, $V_{S_{H R}}>V_{S_{H G}}$. At low pressures the crack distribution is more important in determining shear velocities: at high pressures cracks are closed, and the effect of mineral anisotropy dominates. Thus, measuring shear velocity under hydrostatic pressure separates crack and mineral anisotropy.

Now consider the same experiment in situ, where the Earth acts as a pressure vessel. Jolly (1956) has measured shear velocities in shale. Shale is a prime example of a rock with both crack and mineral anisotropy. Jolly found a large difference (50 to 100 per cent) in $S H$ and $S V$ velocities at shallow depths; the difference decreased at greater depth. To interpret these results, consider the effect on cracks and mineral layering due to the increase in pressure with depth in the Earth. Cracks close with depth (pressure), but mineral layering is unaffected. The large difference between $S H$ and $S V$ velocities near the surface is due mainly to dense concentration of horizontally oriented cracks. With depth the cracks close, and the difference between $S H$ and $S V$ velocities approaches the value expected solely for mineral layering. Thus, because the Earth provides a graded 
pressure scale with depth, we can carry out the same experiment in situ that we did with Chelmsford granite in the laboratory. We can, therefore, separate the sources of anisotropy.

It follows then that anisotropy for a given lithological unit which varies with depth is crack a nisotropy. In the same way, anisotropy which does not vary with depth is mineral anisotropy. Finally, any anisotropy at depths where pressure is greater than 1 or $2 \mathrm{~kb}$ is certainly mineral anisotropy, for, at such pressure, all cracks are closed.

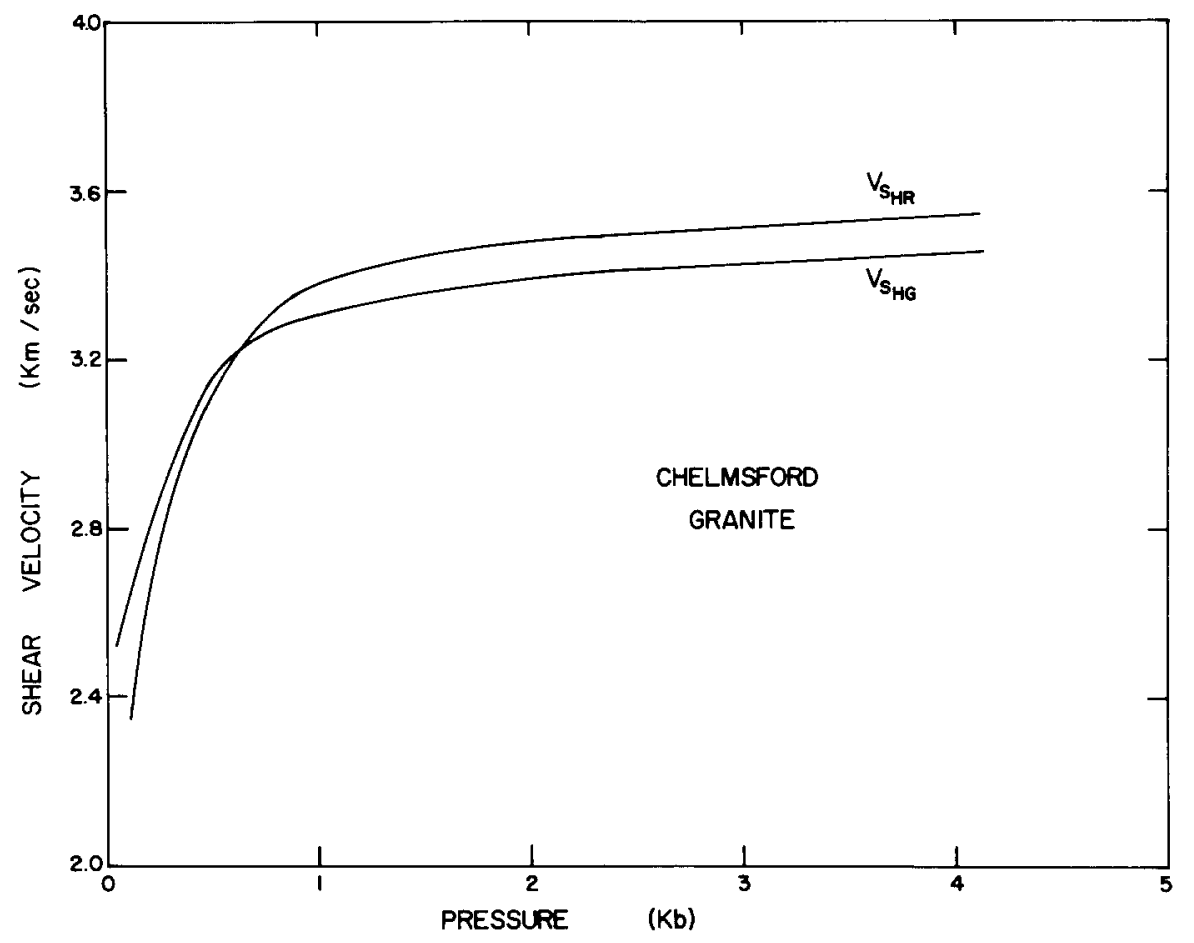

FIG. 3. Shear velocity normal to the headgrain plane as a function of pressure to $5 \mathrm{~kb}$ in the Chelmsford granite. Velocity profiles are shown for transducer polarization normal to the grain $\left(V_{S_{H G}}\right)$ and rift $\left(V_{S_{H R}}\right)$ planes.

\section{CONCLUSIONS}

Previously, most authors (e.g., Simmons, 1964) have reported only one shear velocity for a given propagation direction in rocks. It has now become evident that a single shear velocity value does not fully describe shear-wave propagation in a given direction for anisotropic rocks. The difference in the two shear velocities for waves having the same propagation path and perpendicular polarizations can exceed 10 per cent. Thus, in rock samples with anisotropy in crack and mineral distribution, more than a single shear velocity measurement must be obtained in each propagation direction in order that the velocities completely describe the elastic behavior of the rock.

Recognizing the fact that shear velocities carry information about anisotropy, crack and mineral anistropy in the Earth's crust and mantle and in the lunar interior can be studied using shear waves. In particular, much information can be obtained from shear velocity measurements of mineral anisotropy in the Earth's upper mantle: mineral layering in terrestrial and lunar volcanic rocks, naturally occurring crack anisotropy in 
granites and shales, and stress-induced crack anisotropy in areas of the Earth's crust under stress.

\section{ACKNOWLEDGMENTS}

We wish to thank Dorothy Richter for her analysis of our rock samples, Herbert Wang for his valuable advice throughout the course of this work, and NASA Contract NGR-22-009-540 for support of this work. We are especially grateful to Andrew Fletcher, H. E. Fletcher Co., North Chelmsford, Massachusetts, for supplying the Chelmsford granite samples.

\section{REFERENCES}

Adams, L. H. and E. D. Williamson (1923). The compressibility of minerals and rocks at high pressure, J. Franklin Inst. 195, 475-529.

Aki, K. (1967). Scaling law of seismic spectrum, J. Geophys. Res. 72, 1217-1231.

Aki, K., T. De Fazio, P. Reasenberg, and A. Nur (1970). An active experiment with earthquake fault for an estimation of the in situ stress, Bull. Seism. Soc. Am. 60, 1315-1336.

Ave'Lallement, H. G. and N. L. Carter (1970). Syntectonic recrystallization of olivine and modes of flow in the upper mantle, Bull. Geol. Soc. Am. 81, 2203-2220.

Backus, G. E. (1962). Long wave elastic anisotropy produced by horizontal layering, J. Geophys. Res. 67, 4427-4440.

Baker, D. W. and N. L. Carter (1972). Seismic velocity anisotropy calculated for ultramafic minerals and aggregates, in Flow and Fracture of Rocks, Geophys. Monograph 16, 157-166, American Geophysical Union, Washington, D.C.

Bentley, C. R. (1972). Seismic wave velocities in anisotropic ice: a comparison of measured and calculated values in and around the deep drill hole at Byrd Station, Antarctica, J. Geophys. Res. 77, 4406-4420.

Birch, F. (1960). The velocity of compressional waves in rocks to 10 kilobars, part 1, J. Geophys. Res. 65 , 1083-1102.

Birch, F. (1961). The velocity of compressional waves in rocks to 10 kilobars, part 2, J. Geophys. Res. 66, 2199-2224.

Brace, W. F. (1965). Some new measurements of linear compressibility of rocks, J. Geophys. Res. 70, 391-398.

Brace, W. F., B. W. Paulding, and C. Scholz (1966). Dilatancy in the fracture of crystalline rock, $J$. Geophys. Res. 71, 3939-3954.

Brekhovskikh, L. M. (1960). Waves in Layered Media, R. T. Beyer, Editor, (transl. by D. Leiberman), Academic Press, New York.

Brothers, R. N. and K. A. Rodgers (1969). Petrofabric studies of ultra-mafic nodules from Aukland, New Zealand, J. Geol. 77, 452-465.

Brune, J. N. (1969). Surface waves and crustal structure, in The Earth's Crust and Upper Mantle, Geophys. Monograph 13, 230-242, American Geophysical Union, Washington, D. C.

Byerly, P. (1934). The Texas earthquake of August 16, 1931, Bull. Seism. Soc. Am. 24, 81-99.

Byerly, P. (1938). The earthquake of July 6, 1934: amplitudes and first motion, Bull. Seism. Soc. Am. 28, $1-13$.

Carter, N. L., D. W., Baker, and R. P. George, Jr. (1972). Seismic anisotropy, flow, and constitution of the upper mantle, in Flow and Fracture in Rock, Geophys. Monograph 16, 167-190, American Geophysical Union, Washington, D. C.

Christensen, N. I. (1966). Elasticity of ultrabasic rocks, J. Geophys. Res. 71, 5921-5931.

Christensen, N. I. (1971). Fabric, seismic anisotropy, and tectonic history of the Twin Sisters Dunite, Washington, Bull. Geol. Soc. Am. 82, 1681-1694.

Christensen, N. I. and R. S. Crosson (1968). Seismic anisotropy in the upper mantle, Tectonophysics 6, 93-107.

Christensen, N. I. and R. Ramananantoandro (1971). Elastic moduli and anisotropy of dunite to 10 kilobars, J. Geophys. Res. 76, 4003-4010.

Crosson, R. S. and N. I. Christensen (1969). Transverse anisotropy of the upper mantle in the vicinity of the Pacific fracture zones, Bull. Seism. Soc. Am. 59, 59-72.

Crosson, R. S. and J. W. Lin (1971). Voigt and Reuss prediction of anisotropic elasticity of dunite, J. Geophys. Res. 76, 570-578.

Eisler, J. D. (1969). Investigation of a method for determining stress accumulation at depth, 2, Bull. Seism. Soc. Am. 59, 43-58. 
Ernst, T. (1935). Olivinknollen der basalte als bruchstuche alter olivinfelse, Nachr. Acad. Wiss. Gottingen, Math-Physik. Kl., N.F., Fachgr. 4, 1, 147-154.

Hearmon, R. F. S. (1961). An Introduction to Applied Anisotropic Elasticity, Oxford University Press, Oxford, $136 \mathrm{pp}$.

Herrin, E. (1969). Regional variations in $P$-wave velocity in the upper mantle beneath North America, in The Earth's Crust and Upper Mantle, Geophys. Monograph 13, 242-246, American Geophysical Union, Washington, D.C.

Hess, H. (1964). Seismic anisotropy in the uppermost mantle under oceans, Nature 203, 629-631.

Horai, K. (1969). Cross-covariance analysis of heat flow and seismic delay times for the earth, Earth Planet. Sci. Lett. 7, 213-220.

Jahns, R. H. (1943). Sheet structure in granites: its origin and use as a measure of glacial erosion in New England, J. Geol. 51, 71-98.

Jolly, R. N. (1956). Investigation of shear waves, Geophysics 21, 905-938.

Jones, R. (1952). A method of studying the formation of cracks in a material subjected to stress, Brit. J. Appl. Phys. 3, 229-232.

Kamb, B. (1972). Experimental recrystallization of ice under stress, in Flow and Fracture of Rocks, Geophys. Monograph 16, 211-241, American Geophysical Union, Washington, D.C.

Kanamori, H. (1970). Recent developments in earthquake prediction research in Japan, Tectonophysics 9 , 291-300.

Keen, C. E. and D. L. Barrett (1971). A measurement of seismic anisotropy in the northeast Pacific, Can.J. Earth Sci. 8, 1056-1064.

Matsushima, S. (1960). Variation of the elastic wave velocities of rocks in the process of deformation and fracture under high pressure, Disaster Prevention Res. Inst., Kyoto Univ., Bull. 32, 1-8.

McEvilly, T. V. (1964). Central U.S. crust-upper mantle structure from Love and Rayleigh wave phase velocity inversion, Bull. Seism. Soc. Am. 54, 1997--2015.

Neumann, F. (1930). An analysis of the S-wave, Bull. Seism. Soc. Am. 20, 15-32.

Nur, A. (1971). Effect of stress on velocity anisotropy in rocks with cracks, J. Geophys. Res. 76, 2022-2034.

Nur, A. and G. Simmons (1969a). Stress-induced velocity anisotropy in rock: An experimental study, J. Geophys. Res. 74, 6667-6674.

Nur, A. and G. Simmons (1969b). The effect of saturation on velocity in low porosity rocks, Earth Planet. Sci. Lett. 7, 183-193.

Paterson, M. S. and L. E. Weiss (1961). Symmetry concepts in the structural analysis of deformed rocks, Bull. Geol. Soc. Am. 72, 841-882.

Peng, S. and A. M. Johnson (1972). Crack growth and faulting in cylindrical specimens of Chelmsford granite, Intern. J. Rock Mech. Min. Sci. 9, 37-86.

Raitt, R. W. (1969). Anisotropy in the upper mantle, in The Earth's Crust and Upper Mantle, Geophys. Monograph 13, 250-256, American Geophysical Union, Washington, D.C.

Raitt, R. W., G. G. Shor, Jr., T. J. G. Francis, and G. B. Morris (1969). Anisotropy of the Pacific upper mantle, J. Geophys. Res. 74, 3095-3109.

Shimozuru, D. (1955). Elasticity of rocks under initial stresses, with special reference to the fracture problem, Bull. Earthquake Res. Inst., Tokyo Univ. 33, 437-450.

Simmons, G. (1964). The velocity of shear waves in rocks to 10 kilobars, 1, J. Geophys. Res. 69, 1123-1130.

Simmons, G. and F. Birch (1963). Elastic constants of pyrite, J. Appl. Phys. 34, 2736-2738.

Skehan, J. W., W. J. Keating, R. J. Robinson, and J. B. LeMasurier (1964). Symposium on quarrying in Massachusetts, in Economic Geology in Massachusetts, O. C. Farguhar, Editor, University of Massachusetts Graduate School, 141-160.

Solomon, S. C. (1972). Seismic wave attenuation and partial melting in the upper mantle of North America, J. Geophys, Res. 77, 1483-1502.

Stoneley, R. (1949). The seismological implications of aeolotropy in continental structure, Royal Astron. Soc. Monthly Notices, Geophys. Suppl. 5, 343-353.

Thill, R. E. (1972). Acoustic methods for monitoring failure in rocks, paper presented at 14th Symposium on Rock Mechanics, Pennsylvania State University.

Thomson, W. T. (1950). Transmission of elastic waves through a stratified solid medium, J. Appl. Phys. 21, 89-93.

Tilmann, S. E. and H. F. Bennett (1972). The use of ultrasonic birefringence as a positive indicator of seismic anisotropy, Abstracts with Programs, Geol. Soc. Am., 1972 Annual Meetings 4, 691.

Tocher, D. (1957). Anisotropy of rocks under simple compression, Trans. Am. Geophys. Union 38, 89-94.

Todd, T. and G. Simmons (1972). Effect of pore pressure on velocity of compressional waves in low porosity rocks, J. Geophys. Res. 77, 3731-3743.

Uhrig, L. F. and F. A. Van Melle (1955). Velocity anisotropy in stratified media, Geophysics 20, 774-779. 
Valorovich, M. P., D. B. Balashov, I. S. Tomashevskaya, and V. A. Pavlogradskii (1963). A study of the effect of uniaxial compression on the velocity of elastic waves in rock samples under conditions of high hydrostatic pressure, Bull. Acad. Sci. USSR, Geophys. Ser. (English Transl.) 8, 728-732.

Verma, R. K. (1960). Elasticity of some high density crystals, J. Geophys. Res. 65, 757-766.

Walker, R. K. (1969). The Palisades sill, New Jersey: a reinvestigation, Geol. Soc. Am., Special Paper 111, pp. 178 .

Walsh, J. B. (1965). The effects of cracks on the compressibility of rocks, J. Geophys. Res. 70, 399-411.

Waterman, P. C. and L. J. Teutonico (1957). Ultrasonic double refraction in single crystals, J. Appl. Phys. 28, 266-270.

Wu, T. T. (1966). The effect of inclusion shape on the elastic moduli of a two phase material, Intern. $J$. Solids Struct. 2, 1-8.

Wyss, M. and P. Molnar (1972). Efficiency, stress drop, apparent stress, effective stress, and functional stress of Denver, Colorado earthquakes, J. Geophys. Res. 77, 1433-1438.

DEPARTMENT OF EARTH AND

Planetary Sciences (T. T. and G. S.)

DiVISION OF GEOLOGY AND

Planetary Sciences (W. S. B.)

Massachusetts Institute of Technology

California Institute of Technology

Cambridge, Massachusetts 02139

Pasadena, California 91109

Manuscript received April 27, 1973 